\title{
Why Traditional Metrics May Not Adequately Represent Ethnic Minority Psychology
}

Perspectives on Psychological Science, 2017, Vol I2(6), II62-II65

(c) The Author(s) 2017

Not the version of record.

The version of record is available at https://doi.org//0.1177//74569161770 9590 journals.sagepub.com/home/pps

\section{Moin Syed'}

University of Minnesota

\begin{abstract}
The first Perspectives on Psychological Science symposium (Sternberg, 2016) on eminence in psychology focused almost exclusively on "traditional" markers of success (e.g., citation counts, awards, grants). In this essay I argue that the context of the research and the context of the researchers are crucial components of ethnic minority psychology. First, I describe structural reasons for why ethnic minority researchers might have less impressive traditional markers of success, then I highlight how the "minority tax" contours the scholarly activities of ethnic minority researchers. Any conceptualization of eminence must take these additional considerations into account.
\end{abstract}

\section{Keywords}

Culture, diversity, scientific methodology

'Department of Psychology, University of Minnesota, Minneapolis, MN, USA

\section{Corresponding Author:}

Moin Syed, moin@umn.edu

Note. This document was typeset using Wiernik's (2019) \#prettypreprint template.

Cite this article as: Syed, M. (2017). Why traditional metrics may not adequately represent ethnic minority psychology.

Perspectives on Psychological Science, I2(6), I 162-1 I65. 10.1 177/1745691617709590 
In February $2016 \mathrm{I}$ attended a special meeting at Arizona State University celebrating the $20^{\text {th }}$ anniversary of the publication of the García Coll et al. (1996) integrative model for developmental competencies in minority children-a highly influential "classic" paper in the field. There were about 100 people in attendance, a good mix of senior and junior scholars, graduate students, and some undergraduate students. At one point the topic turned to advocacy and its place in ethnic minority psychology. One of the presenters spoke about the importance of using our research to work with marginalized populations, at which point an audience member yelled out, "after tenure!" The speaker repeated, "of course, after tenure" and many of the senior heads in the room nodded emphaticallyand instructively-to those more junior in the field. As the meeting continued, it became clear that not everyone shared this view. In particular, many of the junior scholars in attendance rejected this advice of engaging in advocacy work "after tenure." For these scholars, advocacy was centrally, even inextricably, linked to their scholarship.

I use this anecdote to suggest that some researchers, particularly those working with ethnic minorities and other marginalized populations, may view their roles as researchers, and therefore "eminence," somewhat differently from others in the field, and certainly differently from the set of papers in the first Perspectives on Psychological Science (PPS) symposium (Sternberg, 2016). Those papers were almost exclusively focused on "traditional" metrics for success (e.g., citation counts, awards, grants), and said little to nothing about how the context of the research or the context of the researchers might be related to those metrics. In this essay, I argue that how eminence should-or last least can-be defined in ethnic minority psychology must take full consideration of both of these issues'.

\section{The Context of the Research: The Value of Descriptive Research.}

There are structural reasons both for why traditional metrics may be depressed for ethnic minority researchers and for why they may be less central for the reward system of such researchers. Science benefits from progressing in a series of steps: I) observe the phenomena of interest, 2) describe the phenomena in detail so as to fully understand its nature, 3) explain the phenomena, largely in terms of causal mechanisms, 4) draw upon an accurate explanation to be able to predict when, and under what conditions, the phenomena will occur, and 5) use prediction accuracy as a basis to control the phenomena. Even a cursory examination of nearly any area of research in psychology will clearly indicate a strong focus on the explanation and prediction phases ${ }^{2}$. Indeed, causal inference and prediction accuracy are the bedrock of mainstream psychological inquiry (Lee, 20I2). It is very difficult to publish purely descriptive research in the top journals in our field, a fact that communicates the implicit (and often explicit) message that if the research does not address causal processes then it does not make a major scientific contribution.

In this context, consider that it is a well-documented fact that psychological research has historically omitted ethnic minorities from its theoretical and research base (Graham, 1992; Hall \& Ma-

\footnotetext{
${ }^{1}$ I use the terms "ethnic minority psychology," "ethnic minority research," and "ethnic minority researchers" somewhat interchangeably. My focal population is ethnic minority researchers conducting ethnic minority psychological research. Some of my points, however, also apply to ethnic majority researchers conducting similar work, as well as ethnic minority researchers not engaged in such work.

${ }^{2}$ A point for another time, but I would argue there is too much emphasis on these phases. In most areas of psychology we have not engaged in sufficient descriptive work so as to properly understand our constructs.
} 
ramba, 200I), and despite strong efforts, not much has changed (Hartmann, et al., 20I3). Indeed, reflecting the ongoing invisibility of this work, as recently as the 2015 Association for Psychological Science (APS) convention, Rob Sellers ${ }^{3}$ was the speaker at a "special event" titled, "Why Should Psychological Science Care About Racial and Ethnic Diversity?" Given the historical and ongoing omission of ethnic minorities in psychological research, there remain substantial gaps in our basic descriptive knowledge of ethnic minority psychology. As a result, researchers with interests in ethnic minority psychology must conduct basic descriptive studies to develop the appropriate knowledge base. This is one reason why there is an abundance of qualitative research with ethnic minorities and other marginalized social groups. Qualitative research is well-suited for gathering data for thick description, leading to a stronger knowledge foundation (Schwab \& Syed, 20I5). But there is also a need for the voices of the silenced to be heard and represented, and qualitative methods are an ideal approach to accomplish this goal.

But this kind of work does not play well to the mainstream of psychology, which is still very much quantitative and focused on trying to understand causality. Funding for ethnic minority research is relatively difficult to come by, because a) it is still not broadly valued by mainstream psychology/science, and b) it is difficult to secure funding for descriptive research, despite the fact that it is essential for further scientific progress. The facts that ethnic minority research is seen as "niche," focuses heavily on description, and that it is more difficult to publish the work in generalist journals all work together to depress citations counts, awards, and the other types of broad recognition championed in the first round of this PPS symposium. Accordingly, these researchers often seek a different type of success, but there is a barrier to doing so.

\section{The Context of the Researchers: Paying the Minority Tax.}

The "minority tax" is a term that has been used to describe how ethnic minority faculty are over-loaded with service requests, particularly for diversity-related service (Rodríguez, Campbell, \& Adelson, 2015). The lack of representation of ethnic minority faculty leads organizations to develop programs, committees, and events to help increase representation. This is a good thing, but ethnic minority faculty are typically recruited for participation in these initiatives, and because they are under-represented, they disproportionately receive such requests. These commitments range across levels of scholarship, from being a voice for minority students on campus, to enhancing diversity initiatives in professional societies, to engaging in editorial duties to break down barriers to publishing research on ethnic minority issues ${ }^{4}$.

As the name implies, the minority tax represents a differential payment of services, one that is not shared to the same extent by most ethnic majority researchers ${ }^{5}$. What needs to be understood about the minority tax, however, it is that it represents a conflict. On the one hand, activities associated with the minority tax can be a drain on one's resources and "take away" from the scholarship

\footnotetext{
${ }^{3}$ This is no dig at Rob, who I greatly admire. Either APS asked him to give this talk, or he chose to do so based on his assessment of what the society needed to hear. Either way, it reflects on APS, not Rob Sellers.

${ }^{4}$ Some may find this difficult to believe, but some journals do explicitly reject research with ethnic minorities simply on the grounds of it being research with ethnic minorities. This has happened to me (Syed, 2017). See also Wang (2016) for a similar experience.

${ }^{5}$ Of course, the minority tax is not always limited to ethnic minorities; faculty with other minority identities (e.g., women, LGBT scholars) can also have similar experiences.
} 
that tends to be rewarded by traditional metrics of eminence. On the other hand, many of these activities are exactly why these researchers are in the field in the first place. Ethnic minority researchers seek to increase the representation, visibility, and recognition of ethnic minorities in psychology. Achieving this goal necessarily involves engaging in service and mentoring that can further diversify the field. Importantly, these efforts may yield no citations, awards, or grant money. For example, collaborating with community organizations to better serve their ethnic minority constituencies can sometimes lead to research projects or opportunities for data collection, but that is not always the case. I have served as a regular consultant for the Science Museum of Minnesota for over five years, working with them to build a more diverse membership base and on developing programs to support STEM interests among ethnic minority youth. I do not have a single citable product that has come from this work. As another example, I frequently give talks and have small meetings with advisors at colleges and universities about considerations when working with ethnic minority students. Similarly, I have conducted workshops with K-I 2 educators, working with them to develop language and tools to better understand their minority students. There is much greater potential for making a difference in people's lives with these activities than if researchers cite my articles. And yet they are not reflected in my $h$-index. Moreover, this important work can have a negative impact on traditional metrics because it diverts time away from writing grants and journal articles.

A limitation to most discussions of the minority tax is that it tends to focus on service activities, but it also has major implications for research activities. Allow me to back up: As described by Stokes (1997) in Pasteur's Quadrant, a central framework for the post-war program of science was the separation of research for knowledge ("basic" research) and research for technological use ("applied" research). Moreover, science was conceptualized as moving linearly, with advancements in knowledge leading to advancements in technology. This can be seen today in the use of the term "translational" research in the social and medical sciences, a term that almost exclusively refers to translations of basic research to applied domains. This way of thinking is so ingrained in $20^{\text {th }}-21^{\text {st }}$ century science that it is largely unquestioned and perhaps even seen as the "natural" way to do science. But this is not so. Stokes (1997) highlights how frequently advances in technology have led to advances in knowledgetranslations the other way. This point echoes Greenwald's (20I2) reformulation of the ubiquitous dictum "there is nothing so practical as a good theory" to "there is nothing so theoretical as a good method." One perhaps counterintuitive set of data that he presented was that Nobel Prizes in science are much more frequently awarded for advances in method as opposed to advances in theory. Thus, despite how we as researchers tend to think science operates, the foundations of inquiry do not require a linear model running from basic to applied research.

In this vein, most ethnic minority research begins with a problem, observed or personally experienced. The motivation for the work is to understand the problem, yes, but also to raise awareness of and ultimately ameliorate the problem. This is true for just about any research, of course, but here we are talking about subordinated populations, ones that do not benefit from institutional power, and so researchers seek to shift that balance of power and bring attention to chronically over-looked issues. Some might claim that this focus represents a conflict of interest, that the researchers who seek to improve the lives of their own ethnic group cannot achieve the objectivity needed to conduct rigorous science. Putting the issue of objectivity aside, this claim is problematic for at least two reasons. First, the same argument does not tend to be applied to researchers who, for example, study Alzheimer's disease because it affected someone in their family (e.g., American Federation for Aging Research, 2017). Indeed, the term "me-search" is often used disparagingly towards 
research that has an autobiographical component. But the reality is that we all conduct me-search in some way-there is always some personal experience that drives the passion necessary to succeed in our work. The question is whether the personal quality is observable by others and openly acknowledged by the researchers. Second, we already know from history that ethnic minority researchers cannot rely on majority group members to advance an inclusive science, and thus the system requires that ethnic minorities pursue such work. If they do not, then nothing will change ${ }^{6}$.

As a result, an often unacknowledged form of the minority tax is an obligation to work for and with the communities that are the subject of the research, with an aim to improve their lives. This approach represents a refusal to treat basic and applied research as separable. Many of my colleagues are involved with policy and social justice efforts that cover a wide variety of issues facing ethnic minorities (e.g., Santos, Menjívar, \& Godfrey, 20I3). These are seen as essential activities, and not something that can "wait for tenure." The work is defined by how it is used in action, and thus any gauge of "eminence" must also take into account the influential activities that surround the work, but do not conform to the traditional methods of assessing success in academia

\section{Conclusion}

To be clear, I am not arguing that ethnic minority researchers are unconcerned with traditional metrics. These indicators - $h$-indexes, citations counts, grant dollars, and so on - are not inconsequential, and they serve as motivators for a great number of researchers. But they must be considered in context, and they are not the only way success is defined. And of course some ethnic minority researchers do embody traditional notions of eminence - Cynthia García Coll and Rob Sellers, mentioned earlier, being two examples. How were they able to do so? There are myriad reasons, but I would argue that a strong reason is that their accomplishments have come about because of the adversity that have experienced, and not despite it (Cooper, 2011). That is, they sought to change what they perceived to be a broken system. And they have inspired countless students to follow their lead. I am reminded of Brian Eno's famous quip about the Velvet Underground, that not many people bought their albums, but everyone who did started a band. There may not be many ethnic minority researchers, but those who are there work hard to have a large impact on the field and the real lives of people.

\section{Declaration of Conflicting Interests}

The author(s) declared no potential conflicts of interest with respect to the research, authorship, and/or publication of this article.

\section{Funding}

The authors received no financial support for the research, authorship, and/or publication of this article.

\section{ORCID iD}

Moin Syed (iD https://orcid.org/0000-0003-4759-3555

\section{References}

American Federation for Aging Research (2017, March 17). Women in science research: Dr. Reisa Sperling. Retrieved from https://www.afar.org/blog/view/women-in-science-research-dr-reisa-sperling.

Cooper, C. R. (2011). Bridging multiple worlds: Cultures, identities, and pathways to college. Oxford University Press.

${ }^{6}$ This point is not meant to negate or minimize the role of majority group allies, of which there are many. Nevertheless, allies alone are not sufficient for progress. 
García Coll, C. G., Crnic, K., Lamberty, G., Wasik, B. H., Jenkins, R., Garcia, H. V., \& McAdoo, H. P. (1996). An integrative model for the study of developmental competencies in minority children. Child Development, 67(5), 1891-1914.

Graham, S. (1992). "Most of the subjects were White and middle class": Trends in published research on African Americans in selected APA journals, 1970-1989. American Psychologist, 47(5), 629.

Greenwald, A. G. (2012). There is nothing so theoretical as a good method. Perspectives on Psychological Science, 7(2), 99108.

Lee, J. J. (2012). Correlation and causation in the study of personality. European Journal of Personality, 26(4), 372-390.

Hall, G. C. N., \& Maramba, G. G. (2001). In search of cultural diversity: Recent literature in cross-cultural and ethnic minority psychology. Cultural Diversity and Ethnic Minority Psychology, 7(1), 12.

Hartmann, W. E., Kim, E. S., Kim, J. H., Nguyen, T. U., Wendt, D. C., Nagata, D. K., \& Gone, J. P. (2013). In search of cultural diversity, revisited: Recent publication trends in cross-cultural and ethnic minority psychology. Review of General Psychology, 17(3), 243-254.

Rodríguez, J. E., Campbell, K. M., \& Adelson, W. J. (2015). Poor representation of Blacks, Latinos, and Native Americans in medicine. Family Medicine, 47(4), 259-263.

Santos, C., Menjívar, C., \& Godfrey, E. (2013). Effects of SB 1070 on children. In L. Magaña \& E. Lee (Eds.), Latino politics and Arizona's immigration law SB 1070 (pp. 79-92). Springer New York.

Schwab, J. R., \& Syed, M. (2015). Qualitative inquiry and emerging adulthood: Meta-theoretical and methodological issues. Emerging Adulthood, 3(6), 388-399.

Sternberg, R. J. (2016). “Am I famous yet?” Judging scholarly merit in psychological science: An introduction. Perspectives on Psychological Science, 11(6), 877-881.

Stokes D. E. (1997). Pasteur's quadrant: Basic science and technological innovation. Washington, DC: Brookings Institution Press.

Syed, M. (2017, April 21). On that time a journal rejected my paper because it was related to culture [Blog post]. Retrieved from http://getsyeducated.blogspot.com/

Wang, Q. (2016). Why should we all be cultural psychologists? Lessons from the study of social cognition. Perspectives on Psychological Science, 11(5), 583-596.

Wiernik, B. M. (2019, October 11). Preprint templates. https://doi.org/10.17605/OSF.IO/HSV6A

\section{Author Biography}

Moin Syed is an Associate Professor of Psychology at the University of Minnesota. His research is focused on identity and personality development among ethnically and culturally-diverse adolescents and emerging adults. He is currently the Editor of Infant and Child Development, is co-Editor (with Kate C. McLean) of the Oxford Handbook of Identity Development, the past Editor of Emerging Adulthood, the official journal of the Society for the Study of Emerging Adulthood, and is past President of the International Society for Research on Identity. 